\title{
Aspectos clínicos e epidemiológicos da epidemia de dengue no Recife, PE, em 2002
}

\author{
Clinical and epidemiological aspects of the \\ dengue epidemic in Recife, PE, 2002
}

\author{
Demetrius Montenegro ${ }^{1,2}$, Heloisa Ramos Lacerda ${ }^{3,4,5}$, Tereza Maciel Lira1, \\ Denise Santos Correia de Oliveira ${ }^{1}$, Ana Antunes Fonseca de Lima ${ }^{1}$, \\ Maria José Bezerra Guimarães ${ }^{1}$ e Petrônio Gusmão de Vasconcelos $^{1}$
}

\begin{abstract}
RESUMO
Este estudo mostra os dados da epidemia de dengue e febre hemorrágica da dengue ocorrida na Cidade do Recife no ano de 2002 e as características clínicas, laboratoriais e de necropsia dos 14 casos de óbito por dengue. Foram notificados 35.597 casos, dos quais 208 foram febre hemorrágica da dengue e 14 evoluíram para óbito. O sorotipo Den-3 ocorreu em $76,3 \%$ dos casos. A maioria dos óbitos ocorreu entre homens com mais de 20 anos, no $11^{\circ}$ dia da doença, assistidos nos hospitais privados. Os valores médios do hematócrito e das plaquetas foram $40,7 \%$ e $56.313 \mathrm{p} / \mathrm{mm}^{3}$, respectivamente. A hepatite, com níveis elevados de transaminases, ocorreu na maioria dos pacientes, que geralmente encontravam-se anictéricos. Dos quatorze casos, 13 tiveram confirmação laboratorial. Em oito casos o óbito decorreu de fenômenos hemorrágicos, entretanto, nos outros seis casos não foram identificados sangramentos significativos. O choque, decorrente do extravasamento vascular, associado ou não a sangramentos significativos, esteve presente em 12 (85,7\%) casos, sendo portanto a principal causa de óbito nos casos graves de dengue.
\end{abstract}

Palavras-chaves: Dengue. Febre hemorrágica da dengue. Necropsia. Óbito.

\begin{abstract}
This paper shows data regarding dengue and hemorrhagic fever of the dengue epidemic in Recife in 2002 and the clinical, laboratorial and necropsy results from the 14 patients who died that year. The sorotype Den-3 was detected in $76.3 \%$ of cases. The majority of deaths occurred among men, over 20 years old, on the $11^{\text {th }}$ day of disease, attended in the private hospitals. The average values of the hematocryt and platelets were $40.7 \%$ and $56,313 \mathrm{p} / \mathrm{mm}^{3}$, respectively. Hepatitis, with high levels of transaminases, occurred in the majority of patients, who generally were anicteric. Of the fourteen deaths, 13 received laboratorial confirmation of the infection. In eight cases death occurred due to hemorrhagic phenomena, however, in the other 6 cases significant bleeding was not identified. Vascular collapse (chock) was present in 12 (85.7\%) cases, with or without the association of major bleeding, and was the most important cause of death.
\end{abstract}

Key-words: Dengue. Hemorrhagic fever of dengue. Necropsia. Death.

Até os meados da década de 50 , a dengue era considerada doença benigna, quando nas Filipinas (1953) surgiu o primeiro surto de dengue hemorrágica (FHD) ${ }^{19}$. Vinte e oito anos mais tarde aconteceu a primeira grande epidemia de FHD das Américas². Desde o início da década de 80, o Brasil vem registrando a circulação simultânea de diferentes sorotipos de vírus da dengue, principalmente os sorotipos Den-1 e Den-2. Em 2001, foi isolado o vírus Den-3, no Estado do Rio do Janeiro ${ }^{10}$ e em 2002, o Brasil estava enfrentando uma explosão de casos de dengue e de FHD ${ }^{23}$. Apesar do elevado número de casos ocorridos recentemente na Ásia, África e Américas a dengue é uma doença cujos mecanismos

\footnotetext{
1. Diretoria de Vigilância Epidemiológica da Secretaria Municipal de Saúde, Recife, PE. 2. Hospital Correia Picanço do Sistema Único de Saúde, Recife, PE. 3. Curso de Pós-Graduação em Medicina Tropical da Universidade Federal de Pernambuco, Recife, PE. 4. Departamento de Medicina Clínica da Universidade Federal de Pernambuco, Recife, PE. 5. Departamento de Medicina Clínica da Universidade de Pernambuco, Recife, PE.

Endereço para Correspondência: Dra. Heloisa Ramos Lacerda de Melo. Rua Prof $\stackrel{a}{a}$ Anunciada da Rocha Melo 97/501, Bairro Madalena, 50710-390 Recife, PE. Tel: 5581 3446-5042; 81 9964-5517.

e-mail: helramos@terra.com.br

Recebido para publicação em 17/5/2005

Aceito em 31/10/2005
} 
fisiopatogênicos que levam ao sangramento, ao choque e até mesmo os achados anatomopatológicos dos casos de óbitos são pouco conhecidos ${ }^{716}$. 0 presente estudo teve como objetivo descrever os dados da epidemia de dengue no Recife em 2002 e analisar os aspectos clínicos, laboratoriais e de necropsia dos pacientes que foram a óbito por dengue nesta epidemia.

\section{MATERIAL E MÉTODOS}

Durante a epidemia de dengue de 2002, a Diretoria de Vigilância Epidemiológica da Secretaria Municipal de Saúde do Recife (DIEVS) teve como fontes notificadoras o disque saúde (central telefônica que servia como fonte notificadora para posterior investigação epidemiológica dos casos), os hospitais públicos e privados (através da Comissão de Controle de Infecção Hospitalar), os laboratórios de análises clínicas privados (mediante envio automático dos resultados dos exames positivos à Secretaria de Saúde do Município) e clínicas privadas, nas quais os médicos realizavam notificação dos casos suspeitos. Como instrumentos de notificação foram utilizadas pelas unidades notificadoras as fichas sinplificadas e as fichas de notificação e investigação do Sistema Nacional de Agravos de Notificação (SINAM) para a dengue. Cada Distrito Sanitário digitava as notificações no banco de dados, dos casos de sua área de abrangência e semanalmente era feita a transferência eletrônica para o DIEVS, onde eram consolidadas as notificações do município. Quando notificados os casos de óbitos por dengue, as cópias dos prontuários eram resgatadas nas unidades de saúde, analisadas juntamente com o resultado dos exames de necropsia, atestado de óbito e com as fichas de notificação, além do registro da visita domiciliar, que era obrigatória. Os autores do estudo compilaram os dados da DIEVS e analisaram os casos de óbito. Os resultados dessas avaliações estão disponíveis neste estudo.

\section{RESULTADOS}

No ano de 2002, ocorreram 35.597 casos de dengue na Cidade do Recife. 0 sexo feminino foi o mais acometido, com 21.857 casos e $80 \%$ dos pacientes tinham mais de 15 anos sendo 208 casos de FHD, com 14 óbitos. A taxa de letalidade por FHD foi de $6,8 \%$. 0 principal sorotipo foi o Den-3, isolado em $76,3 \%$ de 59 pacientes testados.

Entre os 14 pacientes que morreram, a maioria era homens (57,2\%), com idade entre 20-59 anos (57,2\%), procedentes de hospitais privados.

Os pacientes passaram em média 4,2 dias sem procurar assistência médica e um paciente faleceu sem assistência, foi 0 óbito domiciliar. 0 internamento durou em média 7 dias. A duração da doença até o óbito foi em média de 11 dias.

Entre os critérios da Organização Mundial de Saúde (OMS) para o diagnóstico de FHD, que são a febre, as manifestações hemorrágicas, a plaquetopenia e a hemoconcentração (manifestada através de hematócrito aumentado em 20\%, derrames cavitários ou hipoproteinemia) ${ }^{2122}$, apenas 5 pacientes preenchiam todos os critérios, sendo a febre e a plaquetopenia os mais freqüentes. Em 7 pacientes não foi realizada a pesquisa completa da hemoconcentração, principalmente a pequisa de derrames cavitários e a dosagem das proteínas séricas (Tabela 1).

Tabela 1 - Positividade dos critérios para o diagnóstico de febre hemorrágica da dengue, utilizando-se os critérios da OMS, dos 14 casos de óbito por dengue, Recife, 2002.

\begin{tabular}{lcc}
\hline Critérios & Número (*) & Percentagem \\
\hline Febre + manifestações clínicas & $14(14)$ & 100,0 \\
Hemorragia & $10(14)$ & 71,4 \\
Plaquetopenia & $12(13)$ & 92,3 \\
Alteração da permeabilidade capilar & $9(13)$ & 69,2 \\
$\quad$ aumento Ht>20\% dos valores & & \\
$\quad$ normais para a idade e sexo & $6(13)$ & 46,2 \\
$\quad$ derrame cavitário & $7(7)$ & 100,0 \\
$\quad$ hipoalbuminemia & $3(5)$ & 60,0 \\
Exame específico positivo & & \\
(sorologia ou isolamento viral) & $13(14)$ & 92,8 \\
\hline
\end{tabular}

(*) total de pacientes avaliados

As manifestações hemorrágicas ocorreram em 10 (71\%) dos pacientes. 0 sítio de sangramento mais freqüente foi o digestivo, seguido do nasal e do pulmonar. A maioria dos que sangraram tiveram dois sítios diferentes de sangramento. 0 derrame cavitário foi investigado em $50 \%$ dos pacientes, sendo positivo em todos os investigados. 0 mais freqüente foi a ascite, seguido de derrame pleural e pericárdico (Tabela 2).

Tabela 2 - Manifestações hemorrágicas e derrames cavitários dos 14 casos de óbito por dengue, Recife, 2002.

\begin{tabular}{|c|c|c|}
\hline Manifestação & Número & Percentagem \\
\hline \multicolumn{3}{|l|}{ Sangramento } \\
\hline Total de casos com sangramento & 10 & 71,4 \\
\hline clinicamente evidente & 8 & 57,2 \\
\hline \multicolumn{3}{|l|}{ Sítio } \\
\hline aparelho digestivo & 8 & 57,2 \\
\hline nariz & 5 & 35,7 \\
\hline pulmões & 4 & 28,6 \\
\hline pele (petéquias) & 3 & 21,4 \\
\hline aparelho urinário & 3 & 21,4 \\
\hline gengiva & 2 & 14,2 \\
\hline \multicolumn{3}{|l|}{ Número de sítios } \\
\hline 2 & 8 & 57,2 \\
\hline 1 & 3 & 21,4 \\
\hline$>2$ & 1 & 7,1 \\
\hline \multicolumn{3}{|l|}{ Derrame cavitário* } \\
\hline Sim & 7 & 100,0 \\
\hline ascite & 5 & 71,4 \\
\hline derrame pleural & 4 & 57,1 \\
\hline derrame pericárdico & 2 & 28,6 \\
\hline \multicolumn{3}{|l|}{ Número de derrames } \\
\hline 1 & 4 & 57,1 \\
\hline 2 & 2 & 28,6 \\
\hline 3 & 1 & 14,3 \\
\hline
\end{tabular}

*investigado com USG em 7 dos 14 pacientes que morreram 
0 hematócrito dos casos sem sangramento foi em média $48,4 \%$ e dos com sangramento foi $37,3 \%$. A contagem de plaquetas esteve abaixo de 100.000 em 13 casos e a média foi de 48.538. Nos pacientes que sangraram as plaquetas foram em média 36.666 e nos que não sangraram foi de 75.250. As transaminases apresentavam-se, em média 5 a 10 vezes o valor normal (Tabela 3).

Dos 14 pacientes, 7 (50\%) foram submetidos à necropsia $\mathrm{e}$ 13 foram assistidos em hospital. 0 choque hipovolêmico esteve presente em $12(85,7 \%)$ casos. Desses pacientes, quatro apresentaram choque sem sinais de sangramento significativo, mesmo após avaliação pela necropsia e oito pacientes apresentavam hemorragia em múltiplos sítios que poderia ter ocasionado o óbito. Ocorreu um óbito por infarto agudo do miocárdio e um óbito por embolia pulmonar após 35 dias de hospitalização (Tabela 4).
Tabela 3 - Alterações laboratoriais nos 14 óbitos por dengue, Recife, 2002.

\begin{tabular}{lr}
\hline Exame & Valores \\
\hline Hematócrito & \\
média & $40,7 \%$ \\
casos sem sangramento & $48,4 \%$ \\
$\quad$ casos com sangramento & $37,3 \%$ \\
Plaquetas & \\
média & 48.538 \\
casos sem sangramento & 75.250 \\
casos com sangramento & 36.666 \\
$<100.000$ & $13 \mathrm{cas} 0 \mathrm{~s}$ \\
$>100.000$ & $1 \mathrm{cas} 0$ \\
Transaminases & \\
AST (média) & $980 \mathrm{UI}$ \\
ALT (média) & $595 \mathrm{UI}$ \\
Bilirrubina total & \\
média & $8,7 \mathrm{mg} / \mathrm{dL}$ \\
\hline
\end{tabular}

Tabela 4 - Quadro clínico dos casos de dengue no momento do óbito, associado aos achados anatomopatológicos e às causas de morte que constam nos atestados de óbito, Recife, 2002.

\begin{tabular}{|c|c|c|c|}
\hline Caso & Quadro clínico & Necropsia & Causa da morte no atestado \\
\hline 1 & sangramento pulmonar, digestivo e gengivorragia & - & septicemia não especificada \\
\hline 2 & choque e parada cardiorespiratória & congestão pulmonar, cerebral e de aparelho digestivo & enterocolite \\
\hline 3 & choque hipovolêmico & hemorragia de múltiplos órgãos & choque hipovolêmico \\
\hline 4 & choque hipovolêmico por hemorragia digestiva & doença hemorrágica não especificada & choque hipovolêmico \\
\hline 5 & $\begin{array}{c}\text { choque hipovolêmico por hemorragia } \\
\text { pulmonar digestiva e urinária }\end{array}$ & $\begin{array}{l}\text { hemorragia de múltiplos órgãos de } \\
\text { etiologia não especificada }\end{array}$ & choque hipovolêmico \\
\hline 6 & choque sem hemorragia & aterosclerose coronariana & infarto agudo do miocárdio \\
\hline 7 & $\begin{array}{l}\text { choque, embolia pulmonar, } \\
\text { sangramento discreto }\end{array}$ & $*$ & $\begin{array}{c}\text { choque obstrutivo, secundário a } \\
\text { embolia pulmonar }\end{array}$ \\
\hline 8 & choque por hemorragia digestiva alta em hepatopata & $*$ & falência de múltiplos órgãos \\
\hline 9 & $\begin{array}{c}\text { choque hipovolêmico por } \\
\text { hemorragia digestiva e urinária }\end{array}$ & * & distúrbio de coagulação \\
\hline 10 & choque sem sangramento & $*$ & choque não especificado \\
\hline 11 & choque sem sangramento & não encontrados pontos de hemorragia & indeterminado \\
\hline 12 & $\begin{array}{l}\text { choque hipovolêmico por } \\
\text { hemorragia digestiva e pulmonar }\end{array}$ & $*$ & choque hipovolêmico \\
\hline 13 & choque, sangramento discreto & * & indeterminado \\
\hline 14 & óbito domiciliar & hemorragia digestiva e pulmonar & hemorragia pulmonar bilateral \\
\hline
\end{tabular}

\section{DISCUSSÃO}

0 ano de 2002, começou com uma explosão epidêmica de dengue, jamais vista no Recife. Os números dão uma dimensão do problema: neste ano foram notificados 35.597 casos, o que corresponde a uma taxa de incidência de 2.502 casos para cada 100.000 habitantes. A soma dos casos dos sete anos anteriores foi de 43.298, (3.043/100.000), o que corresponde a uma diferença de apenas 1,2 vezes mais casos registrados durante todo o período do que no ano de $2002^{91718}$.

Até o ano de 1997, apenas o sorotipo 1 tinha sido isolado na cidade do Recife. De 1998 em diante, foram isolados dois sorotipos, o Den-1 e o Den-2. No ano de 2002, foram detectados os três sorotipos da dengue na cidade do Recife, os sorotipos 1,2 e 3. Este último foi o mais freqüente 76,3\% ( 45 casos), que também foi isolado das vísceras de dois pacientes que morreram ${ }^{917}{ }^{18}$. Apesar do isolamento de dois sorotipos diferentes, em um mesmo ano, ter ocorrido em 1998, os casos de FHD já tinham sido diagnosticados em duas pessoas em 1996 (0,15/100.000). Os casos de FHD foram aumentando progressivamente para 9 casos em 1997 (0,67 por 100.000$), 37$ casos em 1998 (2,73 por 100.000 habitantes), quando foi registrado o primeiro óbito, o que correspondeu a 2,7\% de letalidade. Em 1999, foram 26 casos de FHD (2,09 por 100.000 habitantes), ano que apresentou a maior letalidade no município, 11,5\% (3 casos). No ano de 2002, apesar do alto coeficiente de detecção de FHD e do maior número de óbitos notificados (14 casos), a taxa de letalidade foi de $6,8 \%$ a segunda mais elevada nos últimos 7 anos $^{1718}$. Observou-se, então, como já ocorrera em Cuba e em Porto Rico, que a epidemia de dengue predominou em adultos $^{314}$, bem como os casos de óbito, diferindo do sudeste asiático, onde os casos graves ocorrem principalmente em crianças com risco até 15 vezes maior de óbito do que nos 
adultos 5 . Apesar de alertadas pelas campanhas de saúde pública, duas pessoas procuraram o serviço relativamente tarde ou não procuraram (quem sabe, não tiveram acesso!), e a média de tempo sem assistência foi de 4,2 dias, semelhante ao estudo porto-riquenho de 90-91, onde as pessoas procuraram serviço médico em torno do quarto e quinto dias de doença ${ }^{15}$. Naquele mesmo país a média de internamento foi de 6,8 dias, enquanto neste estudo foi de 7 dias, muito semelhante. Ao contrário de outros relatos, em que $100 \%$ dos pacientes tiveram algum tipo de manifestação hemorrágica ${ }^{14}$, nesta série de 14 casos, apenas 10 sangraram, o que ocorreu no $4^{\circ}$ dia de doença. Realmente, 0 período mais crítico da doença é o da defervescência da febre, quando podem surgir os sinais de falência circulatória e as manifestações hemorrágicas, o que ocorre em torno do terceiro e quarto dias da doença ${ }^{11}$. Em revisão de 1998, Gubler afirma que as petéquias são a forma mais comum de sangramento na dengue hemorrágica ${ }^{4}$, mas na Índia, o sangramento do trato gastrointestinal $(34,5 \%)$ foi mais frequiente do que as petéquias $(23,6 \%)$ e o sangramento pulmonar $(13,6 \%)^{21}$, coincidindo com os dados deste estudo. A plaquetopenia abaixo de 100.000 esteve presente em 92,3\% dos casos, observando-se uma diminuição do valor de plaquetas de cerca de duas vezes nos pacientes que sangraram comparados aos que não sangraram, apesar de um estudo sugerir que a contagem de plaquetas não seria preditiva do risco de sangramento ${ }^{8}$.

Apenas cinco pacientes completavam os critérios de FHD. Alguns critérios não foram avaliados. 0 maior problema ocorreu com a detecção da hemoconcentração, que dependia de exames complementares como os de imagem para a avaliação de derrames cavitários, que só foi realizado em $50 \%$ dos casos, seja por dificuldade de acesso a ultrassonografia ou pela gravidade do paciente e dificuldade de deslocamento. Soma-se ainda a dificuldade para a avaliação do hematócrito, pois não se conhece o hematócrito basal e os sangramentos e mesmo a reposição volêmica de urgência podem modificar esse parâmetro, como ocorreu neste estudo, pois nos casos que sangraram este encontrava-se com valores normais. Questionase, assim, que a definição clínica de FHD determinada pela OMS, pode não estar apropriada para todos os pacientes ${ }^{613}$.

A realização do estudo necroscópico teve grande importância epidemiológica e clínica. 0 isolamento viral em dois casos foi obtido pela retirada de fragmentos de vísceras. A identificação de alguns sítios de hemorragia também foi conseguida apenas por conta da necropsia. Observou-se a falta de um maior detalhamento sobre a presença de derrames cavitários. As necropsias foram realizadas sem um protocolo prévio, provavelmente reduzindo a atenção para a identificação dessa alteração. Comparando os achados das sete necropsias desse estudo com os dos casos necropsiados em Cuba em 1981, observou-se que a hemorragia digestiva foi a mais freqüente nos dois estudos $(57,2 \%$ e $98 \%$, respectivamente). A hemorragia pulmonar foi encontrada em 43\% dos casos recifenses contra $68 \%$ dos cubanos. E, enquanto aqui não foram relatados derrames cavitários, em Cuba encontrou-se $88 \%$ de casos de ascite, $70 \%$ de casos de efusão pleural e $20 \%$ de derrame pericárdic $0^{12}$.
A morte esteve frequientemente associada à instabilidade hemodinâmica e choque, apesar de ser decorrente de sangramento significativo em apenas $57 \%$ desses pacientes. Mesmo no paciente que morreu por infarto, o extravasamento vascular e a hipotensão provavelmente tiveram papel significante no desencadeamento ou má evolução da doença. Assim a idéia de que os pacientes morreriam de sangramento e que a transfusão de plaquetas teria papel fundamental no tratamento dos pacientes com plaquetopenia parece representar apenas parte do que acontece com os doentes graves. 0 presente estudo é mais uma evidência que os mecanismos imunopatogênicos determinantes do extravasamento vascular precisam ser melhor compreendidos e combatidos pois certamente representam 0 principal mecanismo de óbito ${ }^{1620}$. Além disso sinaliza que a hipotensão deve ser considerado um sinal de alarme e de risco de morte, independente da presença de hemorragias significantes.

\section{REFERÊNCIAS BIBLIOGRÁFICAS}

1. Baluna R, Vitetta ES. Vascular leak syndrome: a side effect of immunotherapy. Immunopharmacology 37: 117-132, 1997.

2. Guzman MG, Kouri G. Dengue and dengue hemorrhagic fever in the Americas: lessons and challenges. Journal of Clinical Virology 27: 1-13, 2003.

3. Guzman MG, Kouri G, Bravo J, Valdes L, Vazquez S, Halstead SB. Effect of age on outcome of secondary dengue-2 infections. International Journal of Infectious Diseases 6: 118-124, 2002.

4. Gubler DJ. Dengue and Dengue Hemorrhagic Fever. Clinical Microbiology Reviews 11: 480-496, 1998.

5. Hongsiriwon S. Dengue hemorrhagic fever in infants. Southeast Asian Journal of Tropical Medicine and Public Health 33: 49-55, 2002.

6. Kalaynarooj S, Vaughn DW, Nimmannitya S. Early and laboratory indicators of acute dengue illness. Journal of Infectious Diseases 176: 313-321, 1997.

7. Lei HY, Yeh TM, Liu HS, Lin YS, Chen SH, Liu CC. Immunopathogenesis of dengue virus infection. Journal Biomedical Science 8: 377-388, 2001.

8. Lum LC, Goh AY, Chan PW, El-Amin AL, Lam SK. Risk factors for hemorrhage in severe dengue infections. Journal Pediatrics 140: 629-631, 2002.

9. Lyra MT. Dengue no Recife: o desafio da epidemia de 2002. In: Mostra nacional de experiências bem sucedidas em epidemiologia, prevenção e controle de doenças. 2, 2002.

10. Nogueira RM, Miagostovich MP, Filippis AM, Pereira MA, Schatzmayr HG. Dengue virus type 3 in Rio de Janeiro, Brazil. Memórias do Instituto Oswaldo Cruz 96: 925-926, 2001.

11. Nogueira S. Dengue. Jornal de Pediatria 75: 9-14, 1999

12. Pan American Health Organization. Dengue and dengue hemorrhagic fever in the Americas: guidelines for prevention and control. Scientific Publication 548: 8, 1994.

13. Phuong CXT, Nhan NT, Kneen R. Clinical diagnosis and assessment of severity of confirmed dengue infectious in vietnamese childen: is the World Health Organization Classification System helpful? American Journal of Tropical Medicine and Hygiene 70: 172-179, 2004.

14. Rigau-Peréz JG. Clinical manifestations of dengue hemorrhagic fever in Puerto Rico: 1990-1991. Revista Panamericana de Salud Publica 1: 381388, 1997.

15. Rigau-Peréz JG, Vorndam AV, Clark GG. The dengue and dengue hemorrhagic fever epidemic in Puerto Rico, 1994-1995. The American Journal of Tropical Medicine and Hygiene 64: 67-74, 2001.

16. Rothman AL, Ennis FA. Immunopathogenesis of Dengue Hemorrhagic Fever. Virology 257: 1-6, 1999. 
17. Secretaria Municipal de Saúde. Diretoria de Epidemiologia e Vigilância à Saúde. Diretoria Executiva de Epidemiologia. Perfil epidemiológico de algumas doenças de notificação compulsória na cidade do Recife. Recife, 2000.

18. Secretaria Municipal de Saúde. Diretoria de Epidemiologia e Vigilância à Saúde. Diretoria Executiva de Epidemiologia. Dengue no Recife: perfil epidemiológico preliminar. Recife, 2002.

19. Sinniah M, Igarashi, A. Dengue Hemorrhagic Fever. Reviews in Medical Virology 5: 193-203, 1995.

20. Tracey KJ, Cerami A. Tumor necrosis factor, other cytokines and disease. Annual Review of Cellular Biology 9: 317-343, 1993.
21. Wali JP, Bswas A, Handa R, Aggarwal P, Wig N, Dwivedi SN. Dengue hemorrhagic in adults: a prospective study of 110 cases. Tropical Doctor 29: 27-30, 1999.

22. World Health Organization. Dengue hemorrhagic fever: diagnosis, treatment, prevention and control, $2^{2}$ edição, p. 1-75, 1996.

23. World Health Organization. Number of reported cases of Dengue and Dengue Hemorrhagic Fever, region of the Americas (by Country and Subregion). Disponível em:http://www.paho.org/english/HCP/HCT/VBD/ dengue-cases-2002 htm>, 2002. 